\title{
VELOCIMETRY FOR MEMS
}

\author{
J.G. Santiago', C.D. Meinhart', S.T. Wereley', D.J. Beebe', and R.J. Adrian ${ }^{3}$ \\ 'Department of Electrical and Computer Engineering \\ and Beckman Institute for Advanced Science and \\ Technology \\ University of Illinois at Urbana-Champaign \\ Urbana, IL 61801 \\ ${ }^{2}$ Department of Mechanical \& Environmental Engineering \\ University of California - Santa Barbara \\ Santa Barbara, CA 93117 \\ ${ }^{3}$ Department of Theoretical and Applied Mechanics \\ University of Illinois at Urbana-Champaign \\ Usbana, IL 61801
}

\begin{abstract}
We have developed a micron-resolution particle image velocimetry (micro-PIV) system to measure instantaneous and ensemble-averaged flow fields in micron-scale fluidic devices. The system images 100-300 $\mathrm{nm}$ diameter seed particles and determines vector fields using a double-frame crosscorrelation algorithm. Velocity fields have been measured with vector-to-vector distances of less than 3.5 microns. This diagnostic tool has achieved the highest velocity measurement resolution to date of an internal fluid flow.
\end{abstract}

\section{INTRODUCTION}

Significant progress has been made in the development of microfluidic devices based on micro-electromechanical system (MEMS) technologies, such as bioanalysis systems, flow sensors, and micro-valves [1-2]. Although the scientific community has witnessed a surge of miniaturization designs, relatively few studies have specifically addressed the physics related to scaling down engineering systems. Since the flow passages of many microfluidic devices have length scales of 1 - 100 microns, traditional flow diagnostic tools cannot be used. Most measurements in microfluidic devices have been limited to bulk properties of the flow. To address this need, we have developed a micron-resolution Particle Image Velocimetry (PIV) system to measure both instantaneous and ensemble-averaged velocity fields in microfluidic devices, with characteristic dimensions of $10-100$ microns. Using this micro-PIV system, we have demonstrated velocity field measurements with 3.5 micron vector-to-vector spacings [3]. Vector spacings of 1 micron are achievable.

\section{EXPERIMENTAL SETUP}

Our system is designed specifically for measuring velocity fields in bioanalysis systems where low-light level imaging is critical. The acquisition system incorporates an epifluorescent microscope with a continuous illumination Mercury-arc lamp, 100 to $300 \mathrm{~nm}$ diameter flow-tracing fluorescent particles, and an intensified CCD camera. To use this system, prototype microfluidic devices must have optical access to the flow field for high numerical-aperture microscope lenses. We have fabricated microfluidic devices by bulk-micromachining [100] Silicon substrate (using a wet $\mathrm{KOH}$ etch) and then sealed the fluid microchannels with a 170 micron thick glass cover slips (see Figure 1). The microchannels are 70 micron wide and 35 micron deep and have the characteristic trapezoidal cross-section of anisotropic [100] etching (no corner compensation was used). The figure also shows a schematic of the microchannel regions we investigated.

\section{RESULTS AND DISCUSSION}

We have used our micro-PIV system to investigate the velocity field of microfluidic devices with characteristic dimensions as low as 10 microns. Figure 2 shows nearlyinstantaneous vector fields obtained in the cross-pattern microchannel shown in Figure 1. These velocity fields were each obtained using two images (of $200 \mathrm{~nm}$ diameter particles) with a center-to-center temporal spacing of $12 \mathrm{~ms}$ and an exposure time of $2 \mathrm{~ms}$. The Reynolds number of these flows (based on channel depth and bulk velocity) is $3 \times 10^{-2}$. The maximum flow velocity in both cases is approximately $1.8 \mathrm{~mm} \mathrm{~s}^{-1}$. The axis values in Figure 2 correspond to the number of CCD pixels. The measurements clearly show the details of the velocity field to within a few microns of the wall. Note that measurement uncertainty due to Brownian motion and image noise is apparent in both images. However, this Brownian motion is uncorrelated in time and space (from particle-to-particle) and can therefore be substantially reduced by either ensemble averaging over several measurements (as discussed below) or by spatial averaging of the instantaneous flow fields.

Figure 3a shows a nearly-instantaneous vector field measurement of a $3 \times 10^{-4}$ Reynolds number Hele-Shaw flow around roughly cylindrical obstruction. Most of the noise in this velocity field measurement is due to the influences of Brownian motion and image noise. Ensemble averaging eight measurements substantially reduces this unbiased noise and yields the velocity data shown in Figure $3 \mathrm{~b}$. The approaching mean velocity is 35 micron $\mathrm{s}^{-1}$ (at the midplane) from the lower left to the upper right. The interrogation spots of the PIV algorithm [4] were $32 \times 32$ pixels with a cross-correlation window offset of six pixels in both the horizontal and vertical directions. Each interrogation spot was overlapped by $50 \%$, producing approximately 900 vectors over a $120 \times 120$ micron field. The spatial resolution is defined by the $6.9 \times 6.9 \times 1.5$ micron interrogation volume (which corresponds to a 3.45 micron vector-to-vector).

\section{CONCLUSIONS}

A micro-PIV technique that can be used to measure velocity fields with vector-to-vector distances of less than 3.5 microns has been demonstrated. This technique combines fluorescence microscopy, intensified CCD imaging, and cross-correlation PIV and can be applied to the study of the flow physics of MEMS-based microfluidic devices. The spatial resolution and accuracy of our systems is ultimately limited by: (1) the diffraction-limited spatial resolution of the imaging system, (2) noise in the particle-image field, (3) the interaction of the fluid with the finite-sized seed particles, and (4) the stochastic influence of Brownian motion. 


\section{REFERENCES}

1. J.P. Brody, P. Yager, R.E. Goldstein, R. H. Austin, "Biotechnology at low Reynolds numbers." Biophys J., 71, 3430-3441, (1996).

2. P. Gravesen, J. Branebjerg, O.S. Jensen , "Microfluidics - A Review," J. of Micromechanics and Microengineering, (1993).

3. Santiago, J.G., S.T. Wereley, C.D. Meinhart, D.J. Beebe, and R.J. Adrian , "A Particle Image Velocimetry System for Microfluidics," in press, Experiments in Fluids (1997).

4. R. J. Adrian, "Particle-imaging techniques for experimental fluid mechanics." Annual Review of Fluid Mechanics, 23, 261-304, (1991).

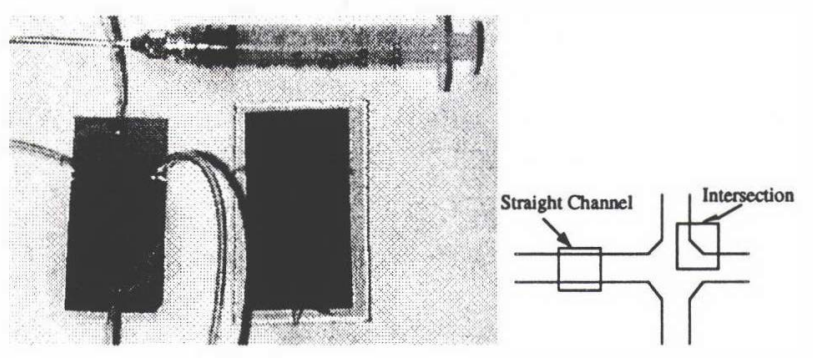

Figure 1. To the left are two sealed cross-pattern microfluidic channels next to a 3 cc syringe. The channels are sealed with a 170 micron thick glass and an RTV adhesive. To the right is a schematic of two regions in the system that were analyzed using the micro-PIV technique.

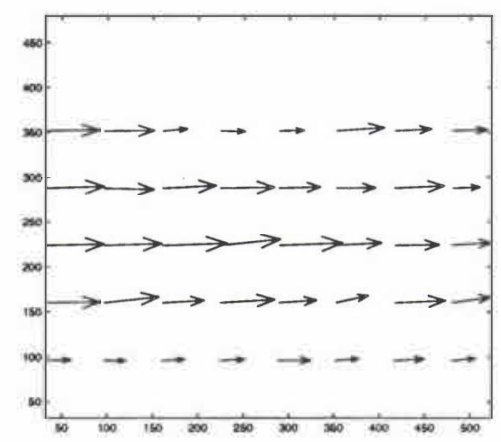

(a)

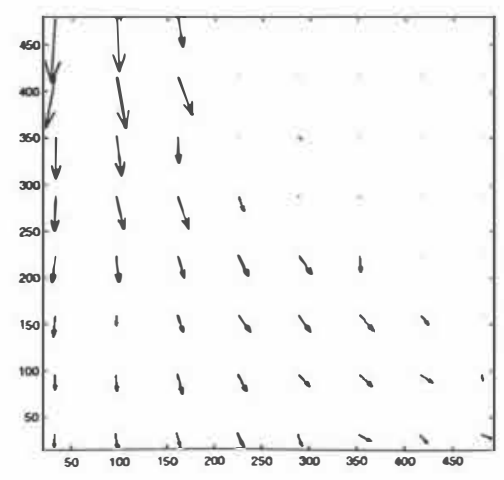

(b)

Figure 2. Velocity field measurements obtained in one of the microfluidic systems shown in Figure 2. Each of these velocity fields were obtained from two particle images. Axis values are number of pixels with 0.23 micron/pixel. (a) Velocity field in a straight section of the cross-pattern microchannel system, (b) Velocity field in the microchannel intersection.

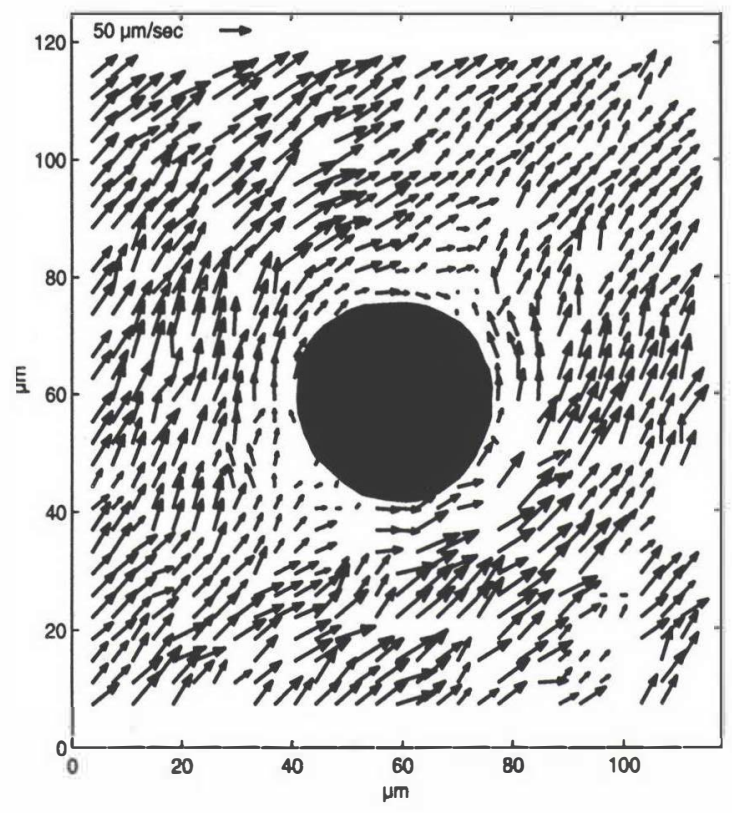

(a)

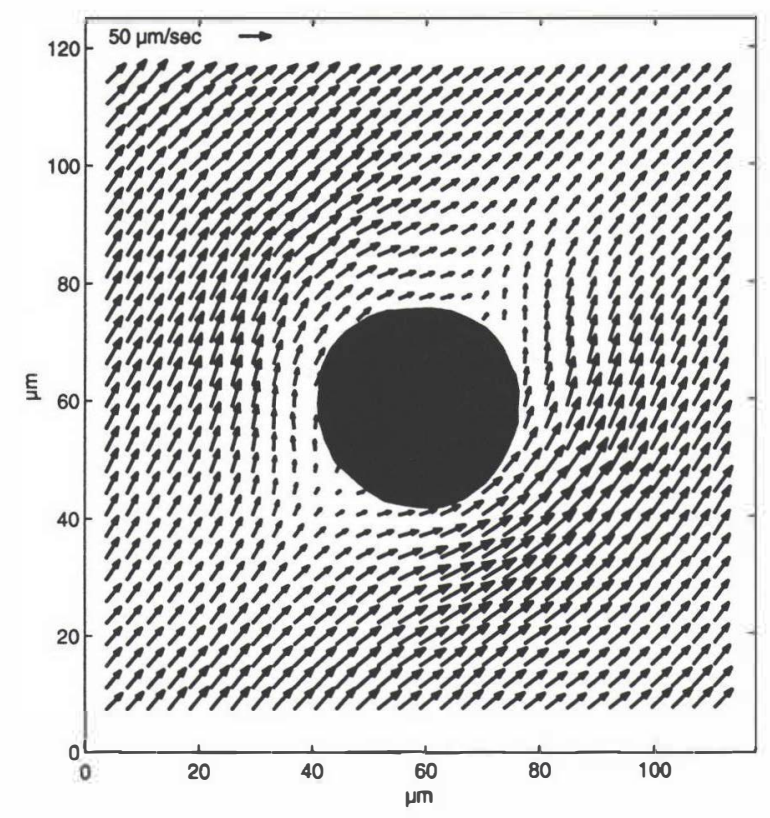

(b)

Figure 3. Vector fields of a surface-tension driven HeleShaw flow around a 30 micron diameter obstacle. Each field contains approximately 900 velocity vectors covering a 120 $x 120$ micron field of view. Each velocity vector was measured with a $6.9 \times 6.9 \times 1.5$ micron measurement volume: (a) Instantaneous vector field measurement, (b) Eight-image ensemble-averaged PIV velocity vector field. 